TITLE:

\title{
Electron acceleration by Z-mode waves associated with cyclotron maser instability
}

$\operatorname{AUTHOR}(S):$

Lee, K. H.; Omura, Y.; Lee, L. C.

\section{CITATION:}

Lee, K. H. ...[et al]. Electron acceleration by Z-mode waves associated with cyclotron maser instability. Physics of Plasmas 2012, 19(12):

122902.

ISSUE DATE:

2012-12-17

URL:

http://hdl.handle.net/2433/187947

\section{RIGHT:}

(c) 2012 American Institute of Physics. This article may be downloaded for personal use only. Any other use requires prior permission of the author and the American Institute of Physics. 


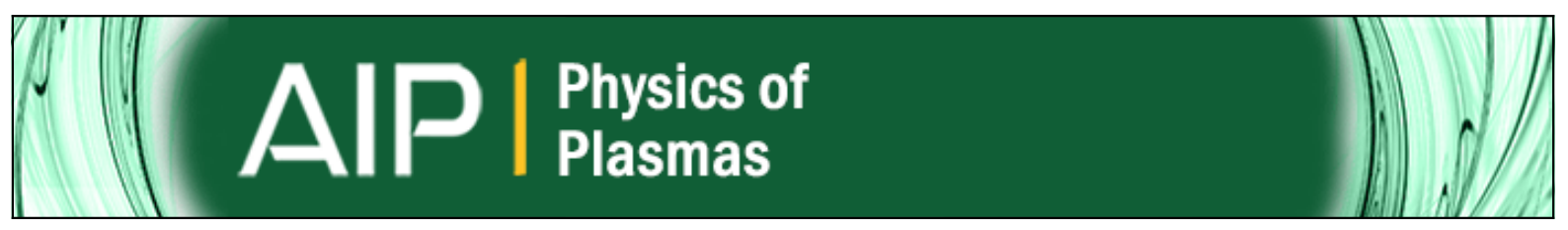

\section{Electron acceleration by Z-mode waves associated with cyclotron maser instability}

K. H. Lee, Y. Omura, and L. C. Lee

Citation: Physics of Plasmas (1994-present) 19, 122902 (2012); doi: 10.1063/1.4772059

View online: http://dx.doi.org/10.1063/1.4772059

View Table of Contents: http://scitation.aip.org/content/aip/journal/pop/19/12?ver=pdfcov

Published by the AIP Publishing

\section{Articles you may be interested in}

Electron acceleration by Z-mode and whistler-mode waves

Phys. Plasmas 20, 112901 (2013); 10.1063/1.4829439

Electron cyclotron maser emission mode coupling to the z-mode on a longitudinal density gradient in the context of solar type III bursts

Phys. Plasmas 19, 110702 (2012); 10.1063/1.4769104

A 2D simulation study of Langmuir, whistler, and cyclotron maser instabilities induced by an electron ring-beam distribution

Phys. Plasmas 18, 092110 (2011); 10.1063/1.3626562

Plasma maser with cyclotron resonance

Phys. Plasmas 4, 3703 (1997); 10.1063/1.872266

Quasilinear evolution of the weakly relativistic electron cyclotron maser instability

Phys. Plasmas 4, 2697 (1997); 10.1063/1.872139

\section{AAP Re-register for Table of Content Alerts}




\title{
Electron acceleration by Z-mode waves associated with cyclotron maser instability
}

\author{
K. H. Lee, ${ }^{1}$ Y. Omura, ${ }^{2, a)}$ and L. C. Lee ${ }^{1, a)}$ \\ ${ }^{1}$ Institute of Space Science, National Central University, Zhongli, Taiwan \\ ${ }^{2}$ Research Institute for Sustainable Humanosphere, Kyoto University, Uji, Kyoto, Japan
}

(Received 7 August 2012; accepted 27 November 2012; published online 17 December 2012)

\begin{abstract}
We demonstrate by a particle simulation that Z-mode waves generated by the cyclotron maser instability can lead to a significant acceleration of energetic electrons. In the particle simulation, the initial electron ring distribution leads to the growth of Z-mode waves, which then accelerate and decelerate the energetic ring electrons. The initial ring distribution evolves into an X-like pattern in momentum space, which can be related to the electron diffusion curves. The peak kinetic energy of accelerated electrons can reach 3 to 6 times the initial kinetic energy. We further show that the acceleration process is related to the "nonlinear resonant trapping" in phase space, and the test-particle calculations indicate that the maximum electron energy gain $\Delta \varepsilon_{\max }$ is proportional to $B_{w}^{0.57}$, where $B_{w}$ is the wave magnetic field. (C) 2012 American Institute of Physics. [http://dx.doi.org/10.1063/1.4772059]
\end{abstract}

\section{INTRODUCTION}

The cyclotron maser instability (CMI) is an important mechanism for wave generation in plasma physics. Wu and $\mathrm{Lee}^{1}$ and Lee and $\mathrm{Wu}^{2}$ discovered the importance of relativistic effect on the resonance condition for the generation of $\mathrm{X}$-mode and O-mode waves. Wagner et al., ${ }^{3}$ Pritchett et al., ${ }^{4}$ and Lee et al. ${ }^{5}$ simulated CMI based on various velocity distributions with population inversion.

In the previous literature, the generation of $\mathrm{X}$ - and O-mode waves was often discussed. ${ }^{1-7}$ The two wave modes are important because they can be directly amplified and propagate into field-free space. The $\mathrm{Z}$ mode needs mode-conversion to generate waves propagating into field-free space. ${ }^{8}$ The whistlermode waves excited by CMI is less discussed. However, the electron acceleration by electromagnetic whistler-mode waves has been widely studied recently. ${ }^{9}$

In our previous simulation study on ring-beam $\mathrm{CMI}{ }^{5}$ we focused on the generation of various wave modes. In this paper, we examine the electron acceleration by carrying out a series of particle simulations with an initial energetic electron ring distribution in the presence of a dense background plasma. The initial electron ring distribution leads to excitation of Z-mode waves and evolves into an X-like pattern in momentum space. Strong acceleration and deceleration of electrons occur, and the peak energy of accelerated electrons can be 3 to 6 times the initial energy, where the initial energy ranges from 100 to $500 \mathrm{keV}$. The $\mathrm{X}$-like pattern is associated with particle diffusion curves. The acceleration process is then studied through test-particle calculations.

\section{EXCITATION OF WAVES AND FORMATION OF X-LIKE PATTERN IN PARTICLE SIMULATIONS}

We use $1 \mathrm{D}$ and 2D electromagnetic particle-in-cell simulation codes (EM codes) ${ }^{10}$ to study the problem. A ring

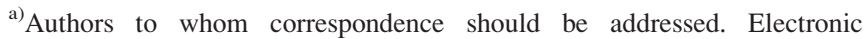
addresses: omura@rish.kyoto-u.ac.jp and loulee@jupiter.ss.ncu.edu.tw.
}

distribution function in the momentum space can be expressed as

$$
f_{r}=n_{r} a_{r} \exp \left[-\frac{\left(u_{\perp}-u_{r \perp}\right)^{2}+u_{\|}^{2}}{2(\Delta u)^{2}}\right],
$$

where $u_{\perp}$ and $u_{\|}$are momentum components perpendicular and parallel to ambient magnetic field $\mathbf{B}_{\mathbf{0}}$, respectively; $u_{r \perp}$ is the ring momentum; $a_{r}$ is a normalization constant. Here, the term "momentum" refers to "momentum per unit mass," $\mathbf{u}=\gamma \mathbf{v}$, where $\gamma$ is the Lorentz factor. The density of the ring distribution $n_{r}$ is set to $5 \%$ of the total electron density. The momentum dispersion of energetic ring electrons is $\Delta u=0.025 c$, and the thermal momentum of background electrons is $0.05 c$, where $c$ is the speed of light. The ratio of electron plasma to cyclotron frequency is $\omega_{p e} / \Omega_{c e} 0.2,0.33,0.5$, and 1 . The average initial kinetic energy, $\varepsilon_{0} \equiv\left(\gamma_{0}-1\right) m_{e} c^{2}$, of ring distribution is set to $50,100,200$, and $500 \mathrm{keV}$ in the simulation, corresponding to initial Lorentz factor $\gamma_{0} \equiv \sqrt{1+u_{r \perp}^{2} / c^{2}}=1.1,1.2,1.4$, and 2, respectively, where $m_{e}$ is the electron rest mass.

Fig. 1(a) shows the dispersion diagrams of $1 \mathrm{D}$ and $2 \mathrm{D}$ simulations for case I $\left(\omega_{p e} / \Omega_{c e}=0.33\right.$ and $\left.\varepsilon_{0}=200 \mathrm{keV}\right)$. From the dispersion diagrams with frequency $\omega$ and parallel wave vector $\mathbf{k}$ in Fig. 1(a), we can see intensive excitation of $\mathrm{R}$-mode waves with frequency between $\omega_{p e}$ and $\Omega_{c e}$. These waves are the Z-mode waves. Fig. 1(b) shows the wave and kinetic energy histories of 1D simulation for case I. Initially waves grow, and most electrons are decelerated. Acceleration occurs as waves reach higher amplitude during the initial linear wave growth stage. The corresponding energetic electron distribution at a later stage is shown in Fig. 2(a) and has high resemblance to the $2 \mathrm{D}$ simulation result. Both $1 \mathrm{D}$ and 2D results show similar dispersion diagrams and similar strong acceleration of electrons with a peak energy $\varepsilon_{p} \approx 3 \varepsilon_{0}=600 \mathrm{keV}$. In the following discussion, we examine the acceleration mechanism by parallel Z-mode waves based on the 1D simulations. 

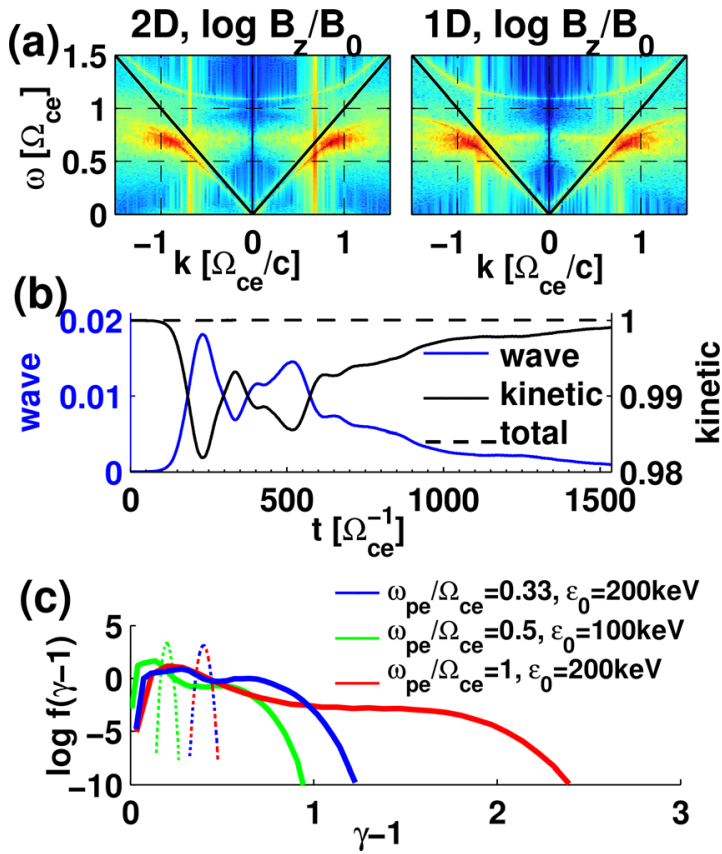

FIG. 1. (a) Dispersion diagrams of $1 \mathrm{D}$ and 2D simulations showing the excitation of Z-mode waves (red color) and (b) time histories of wave, kinetic and total energies in $1 \mathrm{D}$ simulation for case I with $\omega_{p e} / \Omega_{c e}=0.33$ and $\varepsilon_{0}=200 \mathrm{keV}$. The V-like lines in (a) indicate the speed of light. Plot (c) shows the energy distributions for three cases. The initial energy distributions are also shown as thin dotted lines (green line for case II, blue-red for cases I and III).

Fig. 1(c) shows the electron energy distribution for three cases with peak energy of accelerated electrons $\varepsilon_{p} \approx 3 \varepsilon_{0}$ $=600 \mathrm{keV}$ for case I, $\varepsilon_{p} \approx 4.55 \varepsilon_{0}=455 \mathrm{keV}$ for case II $\left(\omega_{p e} / \Omega_{c e}=0.5\right.$ and $\left.\varepsilon_{0}=100 \mathrm{keV}\right)$, and $\varepsilon_{p} \approx 6 \varepsilon_{0}=1200 \mathrm{keV}$ for case III $\left(\omega_{p e} / \Omega_{c e}=1\right.$ and $\left.\varepsilon_{0}=200 \mathrm{keV}\right)$. The peak energy can reach about 3 to 6 times the initial energy.

Figs. 2(a) and 2(b) show the X-like and shell patterns found, respectively, in case I and in the case with $\omega_{p e} / \Omega_{c e}=5$ and $\varepsilon_{0}=200$, where the whistler mode dominates in the latter case. The difference between the diffusive patterns can be related to the phase velocity $v_{p h}$ of the dominant counter-streaming waves and the corresponding electron diffusion curves. The diffusion curve is determined by the conservation of kinetic energy in the frame of reference moving with $v_{p h}{ }^{11} \mathrm{Fig}$. 2(c) shows the growth rate $\omega_{i}$ and $v_{p h}$ of the most unstable wave as functions of $\omega_{p e} / \Omega_{c e}$ obtained from linear kinetic theory (see e.g., Ref. 7) for $\varepsilon_{0}=200 \mathrm{keV}$. The waves leading to the X-like and shell patterns in Figs. 2(a) and 2(b) have $v_{p h} \approx 0.8$ (blue dot) and 0.1 (red dot), respectively. Their corresponding diffusion curves are plotted in Fig. 2(d) as blue and red curves and resemble the simulation results in Figs. 2(a) and 2(b).

\section{TEST-PARTICLE CALCULATIONS}

To further analyze the acceleration and deceleration processes, we conduct a series of test-particle calculations. The equations of electron motion is

$$
m_{e} \frac{d(\gamma \mathbf{v})}{d t}=-e\left[\mathbf{E}_{w}+\mathbf{v} \times\left(\mathbf{B}_{0}+\mathbf{B}_{w}\right)\right]
$$

where $\mathbf{B}_{w}=\mathbf{k} \times \mathbf{E}_{w} / \omega$ with $\mathbf{k} \| \mathbf{B}_{0}$ and $\mathbf{E}_{w} \perp \mathbf{B}_{0}$; $-e$ is the electron charge. Let $\phi$ be the gyration angle of $\mathbf{v}_{\perp}, \psi$ be the gyration angle of $\mathbf{B}_{w}$, and $\zeta \equiv \phi-\psi$ be the gyration angle between $\mathbf{v}_{\perp}$ and $\mathbf{B}_{w}$, as illustrated in Fig. 3. We have

$$
\begin{gathered}
\frac{d \phi}{d t}=\frac{\Omega_{c e}}{\gamma}+\eta, \\
\frac{d \psi}{d t}=\left(\omega-k v_{\|}\right), \\
\theta \equiv \frac{d \zeta}{d t}=\frac{\Omega_{c e}}{\gamma}-\left(\omega-k v_{\|}\right)+\eta \equiv \chi+\eta, \\
\frac{d \gamma}{d t}=-\frac{e}{m_{e} c^{2}} \mathbf{v}_{\perp} \cdot \mathbf{E}_{w}=\left(\frac{e E_{w}}{m_{e}}\right)\left(\frac{v_{\perp}}{c^{2}}\right) \sin \zeta, \\
\chi \equiv \frac{\Omega_{c e}}{\gamma}-\left(\omega-k v_{\|}\right), \\
\eta \equiv \frac{e}{m_{e} \gamma v_{\perp}}\left(E_{w}-v_{\|} B_{w}\right) \cos \zeta .
\end{gathered}
$$
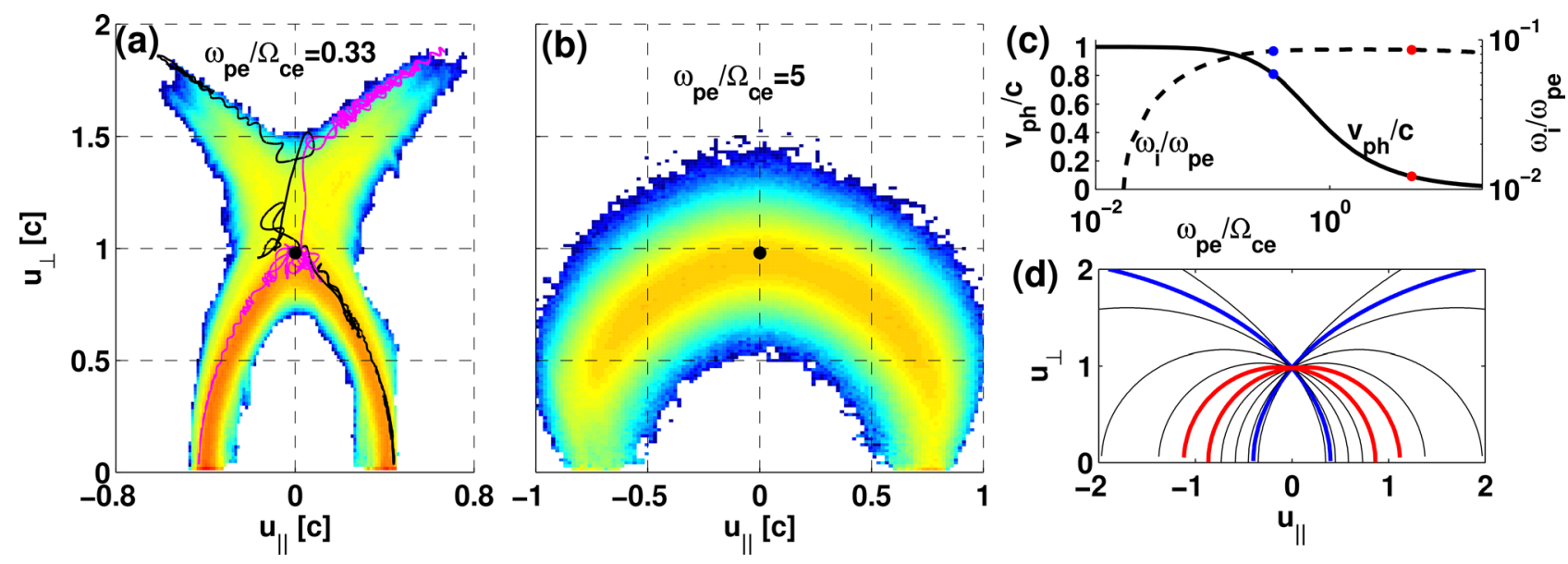

FIG. 2. (a) and (b) Distribution functions of different cases at a later stage obtained from simulations, (c) theoretical calculation of growth rate $\omega_{i}$ and phase velocity $v_{p h}$ of the most unstable mode as functions of $\omega_{p e} / \Omega_{c e}$ and (d) diffusion curves for various $v_{p h}$. In (a) and (b), the black dots indicate the initial electron momentum, and several electron trajectories obtained from EM-code simulation (black lines) and test-particle calculation (purple lines) are superposed. In (c) and (d), the blue dots/curves correspond to the case in (a), and the red ones correspond to (b). 
(a) Forward propagation

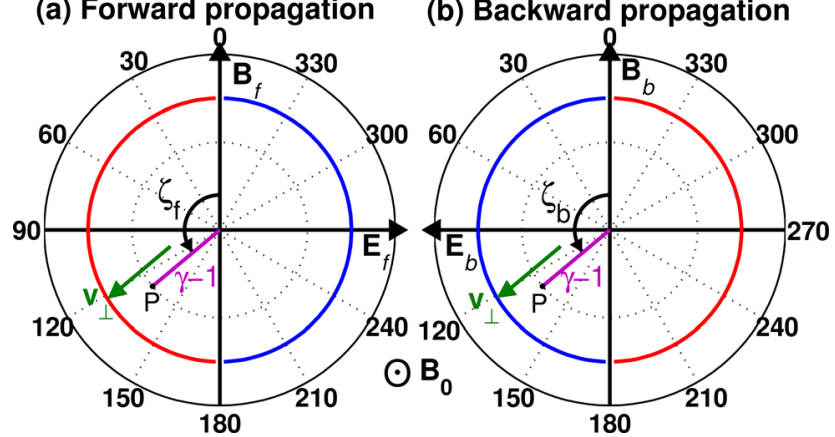

FIG. 3. An illustration of wave fields with forward (f) and backward (b) propagation acting on an electron in the $(\gamma-1, \zeta)$ polar coordinates of magnetic/electric field frame. $\mathbf{E}_{f}$ and $\mathbf{E}_{b}$ are wave electric fields; $\mathbf{B}_{f}$ and $\mathbf{B}_{b}$ are wave magnetic fields. Electron $\mathrm{P}$ has a kinetic energy $(\gamma-1) m_{e} c^{2}$, and its $\mathbf{v}_{\perp}$ makes an angle $\zeta$ with $\mathbf{B}$. The red and blue curves correspond to the acceleration $(d \gamma / d t>0)$ and deceleration phases $(d \gamma / d t<0)$, respectively.

The term $\Omega_{c e} / \gamma$ is associated with electron gyration in the background field $\mathbf{B}_{0}$, and the term $\eta$ is associated with gyration caused by wave fields $\mathbf{E}_{w}$ and $\mathbf{B}_{w}$. The energy increasing rate is expressed in Eq. (6). Usually $\eta$ is small for large $u_{\perp}$ and small wave amplitude. However, $\eta$ can dominate when $u_{\perp}$ is small for electron deceleration case.

\section{ACCELERATION BY A SINGLE WAVE}

In the following, we first analyze single-wave cases to understand the basic acceleration process, and then introduce two-wave cases to explain electron trajectories found in particle simulations.

In a single-wave case, the wave field acting on an electron can be easily understood in $(\gamma-1, \zeta)$ polar plot of Fig. 3 . The variation of kinetic energy $(\gamma-1) m_{e} c^{2}$ is associated with wave electric field. For a forward propagating wave, the electron is accelerated if $0<\zeta<\pi$ (acceleration phase) or decelerated if $\pi<\zeta<2 \pi$ (deceleration phase). An ideal acceleration is that an electron is in the acceleration phase with $\dot{\zeta}=d \zeta / d t=0$ all the time. Nevertheless, the wave field changes electron velocities, $\mathbf{v}_{\perp}$ and $\mathbf{v}_{\|}$, and hence modifies $\chi$ as well as $\eta$, resulting in $d \zeta / d t \neq 0$, and make the electron move out of the acceleration phase after some time. However, an effective acceleration can occur if the electron stays long in the acceleration phase.

We first consider that an electron interacts with a forward propagating wave (single-wave) with constant $E_{w}$ and $B_{w}$. The initial condition is $\zeta=\pi, v_{\|}=-0.257 c, \gamma_{0}=1.4(200 \mathrm{keV})$, $\Omega_{c e} / \gamma_{0}=1.134 \omega, c k / \omega=1.238$, and $B_{w}=0.02 B_{0}$. The purple curves in Fig. 4 are the trajectories of this electron. The electron first rotates in the clockwise $(\mathrm{CW})$ direction (Phase 1) in Fig. 4(a), then the rotation slows down with a gyration reversal (Phase 2), and then this electron rotates in the counterclockwise (CCW) direction (Phase 3). The occurrence of gyration reversals in the magnetic/electric field frame can keep electrons in the acceleration phase longer and leads to a considerable energy gain for electrons. As a result, the electron has an energy gain of $510 \mathrm{keV}$ or $\varepsilon_{p} \approx 3.57 \varepsilon_{0}=710 \mathrm{keV}$ as shown in Fig. 4(c).

Phase 1: Initially, $\eta$ is small and negligible, and we have $\chi<0$ due to negative parallel velocity, leading to the decrease of $\zeta$. The electron rotates in the $\mathrm{CW}$ direction and moves into
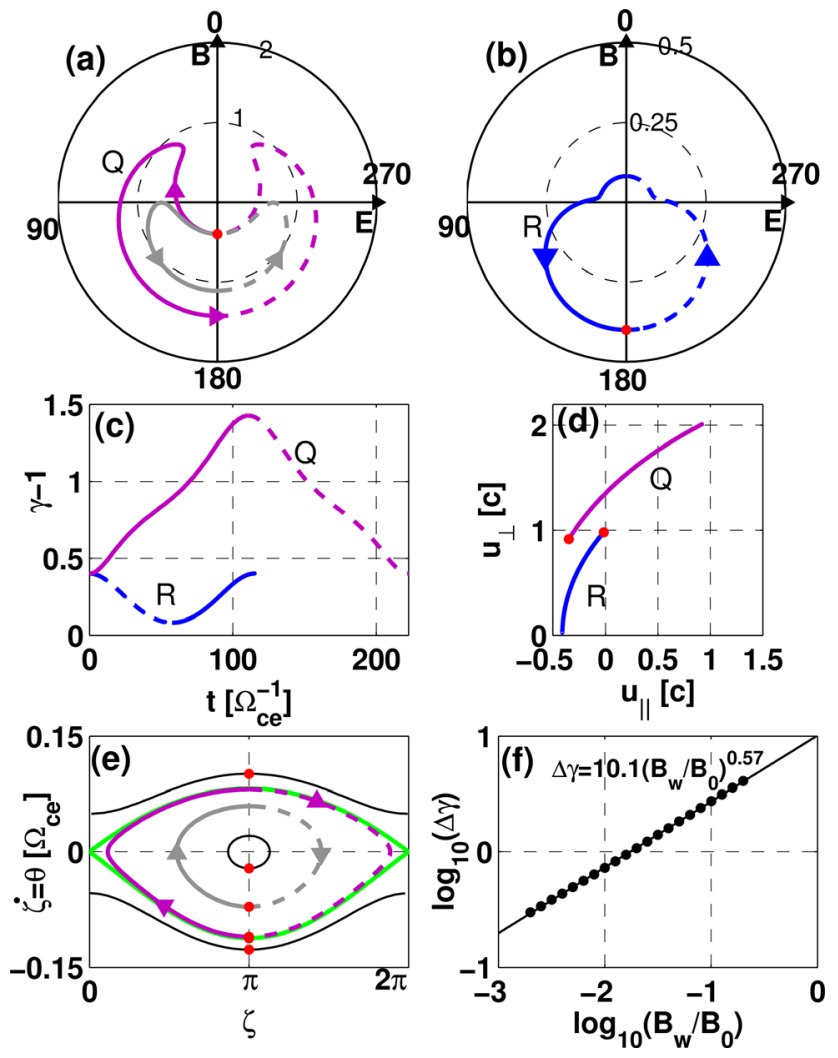

FIG. 4. Single-wave test-particle results for two electrons, Q (purple) and R (blue), with (a)(b) the trajectories in the $(\gamma-1, \zeta)$ coordinates, (c) kinetic energy histories and (d) trajectories in momentum space. Electron Q has one gyration reversal in the acceleration phase of the polar plot and has a peak energy $\varepsilon_{p}=(\gamma-1) m_{e} c^{2} \approx 710 \mathrm{keV}$. Electron $\mathrm{R}$ is decelerated and then accelerated as shown in (c). The electron trajectories in $(\dot{\zeta}, \zeta)$ phase space is shown in (e). The green curve corresponds to the separatrix which gives the maximum energy gain $\Delta \varepsilon_{\max }$. Dashed curves indicates deceleration. The red dots are the initial positions. (f) shows the maximum energy gain $\Delta \varepsilon_{\max }=\Delta \gamma m_{e} c^{2} \approx 10.1 m_{e} c^{2}\left(B_{w} / B_{0}\right)^{0.57}$ for $\gamma_{0}=1.4$.

the acceleration phase. Before the reversal, $d \zeta / d t$ is dominated by $\omega-k v_{\|}$and $\Omega_{c e} / \gamma$. The ratio of their time derivatives is always larger than 1 and almost constant (about 1.6) in both acceleration and deceleration phases, indicating that $\Omega_{c e} / \gamma$ decreases and increases slower than $\omega-k v_{\|}$does.

Phase 2: In the acceleration phase, both $\Omega_{c e} / \gamma$ and $\omega-k v_{\|}$ decrease, and the latter decreases faster, leading to the increase of $d \zeta / d t$, which are initially negative. The value of $d \zeta / d t$ reaches zero at the reversal point before $\zeta=0$ and becomes positive after the reversal. Here, $\chi$ dominates the value of $d \zeta / d t$, and $\eta$ has no major impact on the acceleration process.

Phase 3: The electron now rotates in the CCW direction, and $\zeta$ increases due to positive $d \zeta / d t$, which keeps increasing because of the increase of $\chi$. The electron is still in the acceleration phase before $\zeta=\pi$ and reaches a peak energy $\varepsilon_{p} \approx 3.57 \varepsilon_{0}$.

Fig. 4(b) shows the deceleration process of an electron with $t=0 \sim 60 \Omega_{c e}^{-1}$, in which the term $\eta$ is found to dominate over $\chi$ with a small $u_{\perp}$. The electron kinetic energy reaches a minimum value of $40 \mathrm{keV}(\gamma-1=0.08)$. The deceleration is so effective that the reversal is unnecessary. Here, the electron is decelerated and then accelerated as shown in Fig. 4(c). 


\section{NONLINEAR RESONANT TRAPPING}

The acceleration process discussed above can be related to the "nonlinear resonant trapping" for whistler and ion cyclotron waves as discussed in Ref. 9. For the electron motion in a single wave field, we obtain from Eqs. (2)-(8)

$$
\begin{gathered}
\frac{d^{2} \zeta}{d t^{2}}-\omega_{t r}^{2} \sin \zeta=0 \\
\omega_{t r}^{2}=\Omega_{c e}\left(\frac{B_{w}}{B_{0}}\right)\left(\frac{v_{\perp} k}{\gamma}\right)\left[1-\left(\frac{\omega}{c^{2} k^{2}}\right)\left(k v_{\|}+\frac{\Omega_{c e}}{\gamma}\right)\right] .
\end{gathered}
$$

Fig. 4(e) shows electron trajectories in $(\dot{\zeta}, \zeta)$ phase space, and the result is similar to a pendulum problem. The purple curve is close to the green separatrix, which would give the maximum energy gain for electron acceleration. Fig. 4(f) shows the maximum energy gain obtained from test-particle calculations, $\Delta \varepsilon_{\max }=\Delta \gamma m_{e} c^{2}$, as a function of wave amplitude $B_{w}$ for $\gamma_{0}=1.4$, in which the best fit is $\Delta \gamma=10.1\left(B_{w} / B_{0}\right)^{0.57}$.

The maximum energy gain $\Delta \varepsilon_{\max }$ can be written as

$$
\Delta \varepsilon_{\max }=2 \int_{\pi}^{0} \frac{-e E_{w} v_{\perp} \sin \zeta}{d \zeta / d t} d \zeta
$$

Assuming constant $\omega_{t r}$ and $v_{\perp}$ for simplicity, we obtain $\theta^{2}=2 \omega_{t r}^{2}(1-\cos \zeta)$ from Eq. (9) and substitute it into Eq. (11) to obtain

$$
\Delta \varepsilon_{\max }=8 e E_{w} v_{\perp} / \omega_{t r} \propto B_{w}^{0.5} .
$$

The approximate formula in Eq. (12) is in a good agreement with the fitting line in Fig. 4(f). The small difference of the power, 0.07 , is due to the weak dependence of $v_{\perp}$ and $v_{\|}$on $E_{w}$ or $B_{w}$.

\section{ACCELERATION BY TWO OPPOSITELY PROPAGATING WAVES}

Let the subscripts $\mathrm{b}$ and $\mathrm{f}$ denote forward and backward propagation, respectively. Consider two waves, propagating in opposite directions and parallel to $\mathbf{B}_{0}$, and assume $\omega_{f}=\omega_{b}=\omega, \quad k_{f}=-k_{b}=k, \quad B_{f}=B_{b}=0.01 B_{0} \quad$ and $E_{f}=E_{b}$. The wave amplitude in each of the two waves is half of that in the single-wave case. We find that $\Omega_{c e} / \gamma$ and $\omega \pm k v_{\|}$dominate in most acceleration cases. The Dopplershift frequencies of the two waves are $\omega-k v_{\|}$(forward) and $\omega+k v_{\|}$(backward). Due to opposite signs of wave vectors, it is hard for an electron to stay within the acceleration phases of both waves for a long time if $v_{\|}$is large.

Figs. 5(a)-5(d) show an acceleration case of two-wave test-particle calculation with the initial condition $\zeta_{f}=0.78 \pi$, $\zeta_{b}=1.45 \pi, \quad v_{\|}=-0.06 c, \quad \gamma_{0}=1.4 \quad(200 \mathrm{keV}), \quad \Omega_{c e} / \gamma_{0}$ $=1.1338 \omega$, and $c k / \omega=1.238$. The electron is initially accelerated by both waves (Phase 1) and then interacts with the forward propagating wave in the way similar to the singlewave case while the backward propagating wave just gives a small modulation to the electron energy and momentum (Phase 2). The electron is accelerated for a long period and has a peak energy $\varepsilon_{p} \approx 3.8 \varepsilon_{0}=760 \mathrm{keV}$ as shown in Fig. 5(c).
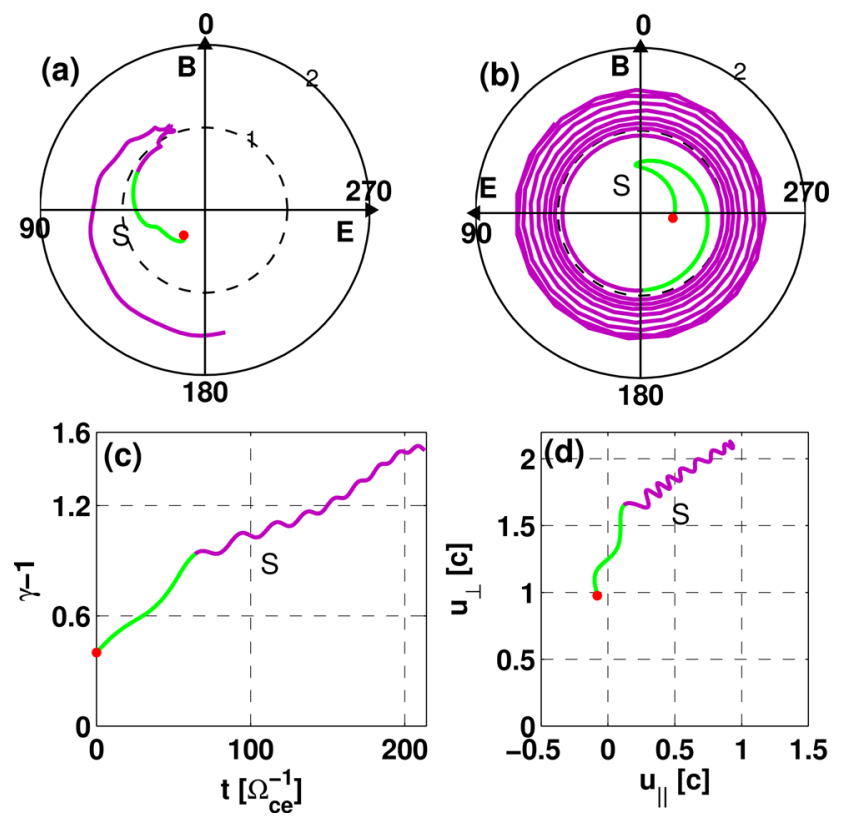

FIG. 5. Two-wave test-particle results for electron S: (a) its trajectory in $\left(\gamma-1, \zeta_{f}\right)$ polar plot, (b) in $\left(\gamma-1, \zeta_{b}\right)$ polar plot, (c) kinetic energy history, and (d) particle trajectory in $u_{\perp}-u_{\|}$momentum space. The red dots indicate the initial positions. Different colors indicate different acceleration stages. The trajectory of electron $\mathrm{S}$ show one gyration reversal, and the peak energy is $\varepsilon_{p} \approx 3.8 \varepsilon_{0}=760 \mathrm{keV}$.

Phase 1 (green curves): At the starting point, we have $\chi_{f} \approx \chi_{b}$ because of small $v_{\|}$. As a result the electron in Phase 1 is accelerated by both the forward and backward propagating waves as denoted by the green curves.

Phase 2 (purple curves): After the initial stage, $v_{\|}$ becomes large, and the difference between $\chi_{f}$ and $\chi_{b}$ also increases. For the forward propagating wave, $\chi_{f}$ increases slowly because both $\Omega_{c e} / \gamma$ and $\omega-k v_{\|}$decrease. The electron can interact with the forward propagating wave in the way similar to the single-wave case. For the backward propagating wave, $\chi_{b}$ decreases rapidly because $\Omega_{c e} / \gamma$ decreases and $\omega+k v_{\|}$increases. Due to a large $\left|\chi_{b}\right|$, the electron gyrates fast in $\left(\gamma-1, \zeta_{b}\right)$ polar plot of Fig. 5(b). As a result of rapid gyration, the backward propagating wave just gives a small modulation to the electron energy and momentum as shown in Figs. 5(c) and 5(d).

In Fig. 2(a), we also show electron trajectories obtained from the EM-code simulation (black lines) and test-particle calculation (purple lines). In the presence of multi-forward and multi-backward propagating waves, electrons take chaotic trajectories as shown in the X-like pattern of Fig. 2(a). Moreover, in Fig. 1(b) the wave energy at the end of the simulation is only $5 \%$ of its peak value. Hence the acceleration process becomes irreversible as wave damping occurs.

\section{DISCUSSION AND SUMMARY}

Electron ring distributions can be produced through several processes, such as mirror reflection of upstream plasma in quasi-perpendicular shocks, ${ }^{7,12}$ mirror reflection of energetic plasma beam in coronal flux loops ${ }^{13}$ and injection of electron beam in the direction perpendicular to magnetic field. ${ }^{14}$ Energization of electrons to $200-2600 \mathrm{keV}$ by Z-mode waves 
may be partially responsible for the observed energetic electrons associated with solar flares and fast magnetosonic shocks in the interplanetary space.

In summary, the energetic electron ring distribution can lead to amplification of Z-mode waves via CMI. Intensive interactions between the parallel propagating Z-mode waves and ring electrons occur, leading to acceleration and deceleration of electrons. The initial ring distribution evolves into an X-like pattern in momentum space, which is related to the electron diffusion curves. Particle simulations show that the peak energy of electrons can reach 3-6 times the initial kinetic energy. The initial kinetic energy in our simulations ranges from 50 to $500 \mathrm{keV}$, while the final peak energy can reach 200 to $2600 \mathrm{keV}$. Reversals of electron gyration in the electric field frame can lead to a longer electron stay in the acceleration phase, resulting in a higher energy gain. We further show that this acceleration process is related to "nonlinear resonant trapping" in $(\dot{\zeta}, \zeta)$ phase space and the maximum electron energy gain $\Delta \varepsilon_{\max }$ is proportional to $B_{w}^{0.57}$ from the test-particle calculations.

\section{ACKNOWLEDGMENTS}

This work was supported by Grant-in-Aid 23340147 of the Ministry of Education, Culture, Sports, Science and Technology in Japan, by the National Science Council in Taiwan (NSC 101-2628-M-001-007-MY3) and by the Center for Computational Geophysics of NCU in Taiwan (CCG Contribution Number: NCU-CCG101-0018).

${ }^{1}$ C. S. Wu and L. C. Lee, Astrophys. J. 230, 621 (1979).

${ }^{2}$ L. C. Lee and C. S. Wu, Phys. Fluids 23, 1348 (1980).

${ }^{3}$ J. S. Wagner, L. C. Lee, C. S. Wu, and T. Tajima, Geophys. Res. Lett. 10, 483, doi:10.1029/GL010i006p00483 (1983).

${ }^{4}$ P. L. Pritchett, R. J. Strangeway, C. W. Carlson, R. E. Ergun, J. P. McFadden, and G. T. Delory, J. Geophys. Res. 104, 10317, doi: 10.1029/1998JA900179
(1999); P. L. Pritchett R. J. Strangeway, R. E. Ergun, and C. W. Carlson, J. Geophys. Res. 107, 1437, doi:10.1029/2002JA009403 (2002).

${ }^{5}$ K. H. Lee, Y. Omura, L. C. Lee, and C. S. Wu, Phys. Rev. Lett. 103, 105101 (2009); K. H. Lee, Y. Omura, and L. C. Lee, Phys. Plasmas 18, 092110 (2011).

${ }^{6}$ L. C. Lee, J. R. Kan, and C. S. Wu, Planet. Space Sci. 28, 703 (1980).

${ }^{7}$ P. H. Yoon, C. B. Wang, and C. S. Wu, Phys. Plasmas 14, 022901 (2007).

${ }^{8}$ R. M. Winglee and G. A. Dulk, Astrophys. J. 307, 808 (1986); D. B. Melrose, Astrophys. J. 380, 256 (1991).

${ }^{9}$ Y. Omura, N. Furuya, and D. Summers, J. Geophys. Res. 112, A06236, doi:10.1029/2006JA012243 (2007); Y. Katoh and Y. Omura, Geophys. Res. Lett. 34, L13102, doi:10.1029/2007GL029758 (2007); N. Furuya, Y. Omura, and D. Summers, J. Geophys. Res. 113, A04224, doi:10.1029/2007JA012478 (2008); M. Hikishima, Y. Omura, and D. Summers, J. Geophys. Res. 115, A12246, doi:10.1029/2010JA015860 (2010); Y. Omura and Q. Zhao, J. Geophys. Res. 117, A08227, doi:10.1029/2012JA017943 (2012); Y. Katoh, Y. Omura, and D. Summers, Ann. Geophys. 26, 3451, doi:10.5194/angeo-26-3451-2008 (2008); D. Summers and Y. Omura, Geophys. Res. Lett. 34, L24205, doi:10.1029/2007GL032226 (2007); Z. Su, F. Xiao, H. Zheng, and S. Wang, J. Geophys. Res. 116, A04205, doi:10.1029/2010JA016228 (2011); D. Summers, B. Ni, and N. P. Meredith, J. Geophys. Res. 112, A04207, doi:10.1029/2006JA011993 (2007); A. Varotsou, D. Boscher, S. Bourdarie, R. B. Horne, N. P. Meredith, S. A. Glauert, and R. H. Friedel, J. Geophys. Res. 113, A12212, doi:10.1029/2007JA012862 (2008).

${ }^{10}$ Y. Omura, "One-dimensional electromagnetic particle code: KEMPO1," in Advanced Methods for Space Simulations, edited by H. Usui and Y. Omura (Terra Sci., Tokyo, 2007), pp. 1-21.

${ }^{11}$ D. Summers, R. M. Thorne, and F. Xiao, J. Geophys. Res. 103, 20487, doi:10.1029/98JA01740 (1998); A. D. M. Walker, Plasma waves in the Magnetosphere (Springer-Verlag, New York, 1993), pp. 151-186.

${ }^{12}$ C. S. Wu, J. Geophys. Res. 89, 8857, doi:10.1029/JA089iA10p08857 (1984); A. O. Benz and G. Thejappa, Astron. Astrophys. 202, 267 (1988); S. Kainer and R. J. MacDowall, J. Geophys. Res. 101, 495, doi: 10.1029/ 95JA02026 (1996).

${ }^{13}$ S. M. White, D. B. Melrose, and G. A. Dulk, Astrophys. J. 308, 424 (1986); C. S. Wu, C. B. Wang, G. C. Zhou, S. Wang, and P. H. Yoon, Astrophys. J. 621, 1129 (2005).

${ }^{14}$ H. P. Freund, H. K. Wong, C. S. Wu, and M. J. Xu, Phys. Fluids 26, 2263 (1983); Y. P. Chen, G. C. Zhou, P. H. Yoon, and C. S. Wu, Phys. Plasmas 9, 2816 (2002) 